alveolar spaces due to alveolar macrophage dysfunction. Autoimmune PAP is driven by circulating autoantibodies against factors involved in macrophage development and maturation. Affected macrophages have both impaired ability to clear surfactant, resulting in abundant surfactant deposition, and defective antimicrobial defence mechanisms, predisposing to atypical lung infections. Cryptococcus gattii is a fungal organism widely present in the environment, and is endemic to tropical and subtropical climate regions, including Australia. Infection is caused by inhalation of fungal spores, and commonly produces a range of clinical manifestations from minimal symptoms to disseminated disease with cryptococcaemia, the outcome of which is determined by a complex set of interacting pathogen and host factors. Cryptococcaemia is rare in those with intact immunity, and this case highlights how PAP and impairment in first-line antimicrobial defence mechanisms likely contributed to the progression to disseminated disease.

\section{DEATH DUE TO TENSION GASTROTHORAX}

Mandy Lau, George Paul

Forensic Medicine Division, Applied Sciences Group, Health Sciences Authority, Singapore

The term 'tension gastrothorax' is used to describe the rare and life-threatening complication of a diaphragmatic defect and can present with non-specific symptoms. It develops when an intrathoracic stomach, which has herniated through a diaphragmatic defect, is distended, causing mediastinal displacement. Here, we present a case report of a previously healthy 2 -year-old boy with tension gastrothorax as well as a review of the literature on this phenomenon. Although this condition is rare, it is an important differential to exclude in children presenting with non-specific symptoms.

\section{GEOMETRIC MORPHOMETRIC STUDY OF SEXUAL DIMORPHISM IN MALAYSIAN MANDIBLES}

Faridah Mohd Nor $^{1}$, Lii Jye Tan ${ }^{1}$, Mohamed Swarhib Shafie ${ }^{1}$, Jessie Hiu

${ }^{1}$ Forensic Unit, Department of Pathology, Universiti Kebangsaan Malaysia Medical Centre, Kuala Lumpur, West Malaysia; and ${ }^{2}$ Department of Forensic Medicine, Queen Elizabeth Hospital, Kota Kinabalu, Sabah, East Malaysia

Background: Geometric morphometric is the statistical analysis of form based on Cartesian landmark coordinates. In this study, geometric morphometric techniques were used to study sexual dimorphism of mandible. Aims: The objectives were to determine mandible variation in size and shape, to determine the relationship between size and shape of mandible in different sexes, and to visualise allometry patterns of mandible.

Methods: About 113 samples of adult human mandibles (65 males, 48 females) were analysed at the Universiti Kebangsaan Malaysia Medical Centre. Approximately 12 landmarks were placed on the 2-D image by landmarking software, and were analysed with MorphoJ.

Results: The first five principal components were taken to represent the morphological variance with $83.64 \%$ cumulative variance. The mandible shape was used to determine sex. The variance visualization showed significant variation between male and female. The classification accuracy was $91.96 \%$.

Conclusion: Geometric morphometric is a good method to study sexual dimorphism of the mandible. Its potential may be developed in forensic anthropology to determine ancestry and age groups. MorphoJ and other softwares may be used in the application of geometric morphometric study of other bones.

\section{AN ANALYSIS OF CHILD AND ADOLESCENT SUICIDE IN THREE CENTRES FROM 2008-2017}

Kelly L. Olds ${ }^{1}$, Rexson Tse ${ }^{2}$, Simon Stables ${ }^{2}$, Andrew M. Baker ${ }^{3}$, Neil E. I. Langlois ${ }^{4,5}$, Roger W. Byard ${ }^{4,5}$ ${ }^{1}$ School of Medicine, The University of Notre Dame Australia, Fremantle, Australia; ${ }^{2}$ LabPLUS, Auckland City Hospital, Auckland, New Zealand; ${ }^{3}$ Hennepin County Medical Examiner, Minneapolis, USA; ${ }^{4}$ School of Medicine, The University of Adelaide, Adelaide, Australia; and ${ }^{5}$ Forensic Science South Australia, Adelaide, Australia

The overall suicide rate worldwide has been found to have changed little over the past 100 years, ${ }^{1}$ but different trends have been observed over time for USA, Australia and NZ. ${ }^{2,3}$ Few studies have focused on child and adolescent ( $<18$ years of age) suicide, meaning there is little prior knowledge from which to determine trends. This project is an update on youth suicides in Adelaide, which were compared with the jurisdictions of Auckland (NZ) and Hennepin County (USA). Youth suicides between 2008-2017 in Adelaide and the other centres were included in this study. Demographic data (age, sex, ethnicity, BMI) and the method of suicide from these three regions were collected and analysed. There were 39 youth suicides in Adelaide (2.4\% of 1661 total suicides), 50 in Hennepin (3.6\% of 1404 total) and 105 in Auckland $(6.4 \%$ of 1638 total) from 2008-2017. The most common method of suicide across all centres was hanging, occurring in $>80 \%$ of cases in Adelaide and Auckland, and $56 \%$ in Hennepin. Hennepin County had a greater proportion of suicides using firearms ( $28 \%$, compared with $1.9 \%$ for Auckland and $5.1 \%$ for Adelaide). Unusual means of suicide were used less frequently by youth than previously.

\section{References}

1. Goldney RD, Harrison J. Suicide in the elderly: some good news. Aust J Age 1998; 2: 54-5.

2. Byard RW, Markopoulos D, Prasad D, et al. Early adolescent suicide: a comparative study. J Clin Forens Med 2000; 7: 6-9.

3. Beautrais AL. Methods of youth suicide in New Zealand: trends and implications for prevention. Aust NZ J Psychiatr 2000; 34: 413-9.

\section{AN UNUSUAL CASE OF HYPOTHERMIA AND PARADOXICAL UNDRESSING IN WESTERN SYDNEY}

Fernando Pisani

Forensic Medical Unit, Blacktown Hospital, Sydney, NSW, Australia

An 83-year-old lady, who was previously well and independent was found by her son in a state of partial undress in the lounge room in her home, after being seen well 1-2 days previously. The back door of the residence was open, some rooms in the house were in disarray, and blood was present in the living room. Police were contacted due to the possibility of an assault. 